\title{
LETTERS
}

\section{Transitioning to outpatient arthroplasty during COVID-19: time to pivot}

The CMAJ research article by Wang and colleagues ${ }^{1}$ highlights the substantial surgical backlog in Ontario that resulted from the first wave of the coronavirus disease 2019 (COVID-19) pandemic. The impact of Ontario's second wave on inpatient resources has exacerbated the surgical backlog even further. The total joint arthroplasty (TJA) program at Sinai Health in Toronto recently introduced an enhanced recovery after surgery bundle, ${ }^{2}$ which has enabled us to pivot quickly to an outpatient TJA program in response to restrictions on inpatient surgery.

After careful review of epidemiologic forecasts and resources that were available early during the pandemic, ${ }^{3}$ our surgical services team anticipated that to continue to be able to perform TJA procedures throughout future waves of the COVID-19 pandemic, we would need to quickly establish a hybrid outpatient/ inpatient arthroplasty program. ${ }^{4}$ An internal needs assessment was performed. Results highlighted the need to develop an evidence-based, patient-centred outpatient pathway. ${ }^{5}$ New interventions included well-defined outpatient selection criteria, modification of anesthetic to facilitate early ambulation, outpatient recovery education materials, and virtual postoperative care follow-up. By January 2021, our program evolved into a hybrid TJA program with more than $25 \%$ of primary referrals for TJA undergoing surgery as outpatients. This outpatient pathway was well-appreciated by patients and hospital leadership alike.

With an output of more than 125000 TJA procedures per year, Canadian arthroplasty centres are faced with the challenge of how to continue providing care during the COVID-19 pandemic. To minimize use of inpatient health resources, hospitals need to "pivot" toward outpatient TJA by rapidly implementing pathways, protocols and resources to ensure that patients undergoing TJA continue to receive the surgical care they need.

Cite as: CMAJ 2021 March 29;193:E455. doi: $10.1503 / \mathrm{cmaj} .78145$

\section{Yehoshua Gleicher MD MSc}

Staff anesthesiologist, Sinai Health, Toronto, Ont.

\section{Sharon Peacock MD BSC (PT)}

Staff anesthesiologist, Sinai Health, Toronto, Ont.

\section{Miki Peer PhD}

Scientific associate, University Health Network, Toronto, Ont.

\section{Jesse Wolfstadt MD MSC}

Orthopedic surgeon, Sinai Health, Toronto, Ont.

\section{References}

1. Wang J, Vahid S, Eberg M, et al. Clearing the surgical backlog caused by COVID-19 in Ontario: a time series modelling study. CMAJ 2020;192:E1347-56.

2. Gleicher Y, Siddiqui N, Mazda Y, et al. Reducing acute hospitalization length of stay after total knee arthroplasty: a quality improvement study. J Arthroplasty 2021;36:837-44.

3. O'Connor CM, Anoushiravani AA, DiCaprio MR, et al. Economic recovery after the COVID-19 pandemic: resuming elective orthopedic surgery and total joint arthroplasty. J Arthroplasty 2020;35:S32-6.

4. Tuite AR, Fisman DN, Greer AL. Mathematical modelling of COVID-19 transmission and mitigation strategies in the population of Ontario, Canada. CMAJ 2020;192:E497-505.

5. Bodrogi A, Dervin GF, Beaulé PE. Management of patients undergoing same-day discharge primary total hip and knee arthroplasty. CMAJ 2020; 192:E34-9.

\section{Competing interests: None declared.}

Content licence: This is an Open Access article distributed in accordance with the terms of the Creative Commons Attribution (CC BY-NC-ND 4.0) licence, which permits use, distribution and reproduction in any medium, provided that the original publication is properly cited, the use is noncommercial (i.e., research or educational use), and no modifications or adaptations are made. See: https://creativecommons.org/ licenses/by-nc-nd/4.0/ 